\title{
Managing Cancer and Living Meaningfully (CALM): A qualitative study of a brief individual psychotherapy for individuals with advanced cancer
}

\author{
Rinat Nissim, Emily Freeman, Chris Lo, Camilla Zimmermann, \\ Lucia Gagliese, Anne Rydall, Sarah Hales, and Gary Rodin
}

Version Post-print/accepted manuscript

\begin{abstract}
Citation Nissim R, Freeman E, Lo C, Zimmermann C, Gagliese L, Rydall A, (published version) Hales S, Rodin G. Managing Cancer and Living Meaningfully (CALM): A qualitative study of a brief individual psychotherapy for individuals with advanced cancer. Palliative Medicine, 2012 Jul; 26(5):713-721. doi: $10.1177 / 0269216311425096$
\end{abstract}

Copyright information Copyright (C) 2011 SAGE Publications. Reprinted by permission of SAGE Publications.

\section{How to cite TSpace items}

Always cite the published version, so the author(s) will receive recognition through services that track citation counts, e.g. Scopus. If you need to cite the page number of the author manuscript from TSpace because you cannot access the published version, then cite the TSpace version in addition to the published version using the permanent URI (handle) found on the record page.

This article was made openly accessible by $U$ of $T$ Faculty.

Please tell us how this access benefits you. Your story matters. 


\section{Full citation and link to final published version:}

Nissim R, Freeman E, Lo C, Zimmermann C, Gagliese L, Rydall A, Hales S,

Rodin G. Managing Cancer and Living Meaningfully (CALM): A qualitative study of a brief individual psychotherapy for individuals with advanced cancer.

Palliative Medicine, 2012 Jul; 26(5):713-721. doi: 10.1177/0269216311425096 [Epub 2011 Oct 31] 
Title: Managing Cancer and Living Meaningfully (CALM): A Qualitative Study of a Brief Individual Psychotherapy for Individuals with Advanced Cancer

Running head: Managing Cancer and Living Meaningfully (CALM)

Authors:

Rinat Nissim, Emily Freeman, Chris Lo, Camilla Zimmermann, Lucia Gagliese, Anne Rydall, Sarah Hales and Gary Rodin

Affiliation:

Department of Psychosocial Oncology and Palliative Care, Princess Margaret Hospital, University Health Network

Accepted: Palliative Medicine

Published online first, October 31, 2011 


\begin{abstract}
Background: Although psychosocial care has been regarded as central to palliative and supportive care, there have been few empirically tested approaches to individual intervention. Aim: The subjective experience of advanced cancer patients receiving a new manualized brief individual psychotherapy, referred to as Managing Cancer and Living Meaningfully (CALM), was examined prior to the initiation of a randomized controlled trial testing the effectiveness of this intervention. Design: Semistructured interviews were conducted with patients who had a diagnosis of advanced cancer, and who underwent the intervention. Setting/Participants: Patients were recruited from a large urban regional cancer center in Toronto, Canada. The 10 interviewees included 7 women and 3 men. All had completed between 3 to 6 CALM sessions prior to the interview. Results: The CALM intervention was associated with profound and unique patient-identified benefits and no patient-identified risks or concerns. Five interrelated benefits of the intervention were identified: 1) a safe place to process the experience of advanced cancer; 2) permission to talk about death and dying; 3 ) assistance in managing the illness and navigating the healthcare system; 4) resolution of relational strain; and 5) an opportunity to "be seen as a whole person" within the healthcare system. These benefits were regarded by participants as unique in their cancer journey. Conclusions: Findings from a qualitative study suggest that the CALM intervention provides substantial benefits for patients with advanced cancer prior to the end of life. Findings informed the development of a randomized controlled trial to evaluate the effectiveness of this intervention.
\end{abstract}

Keywords: Cancer, Oncology, Psychotherapy, Advanced cancer, Interviews 
Managing Cancer and Living Meaningfully (CALM):

A Qualitative Study of a Brief Individual Psychotherapy for Individuals with Advanced

Cancer

\section{Introduction}

Psychological well-being is inevitably challenged in individuals with advanced cancer because of the profound and diverse stresses and burdens associated with it. These invariably include physical suffering and disability, the threat of impending mortality, dramatic alterations in support needs and personal relationships, and the challenge of navigating a complex health care system and making treatment decisions that have life and death implications $[1,2]$. While the primary task at the end of life may be to face death with comfort, dignity and a sense of meaning [3, 4], those with a longer expected survival from advanced disease must navigate the simultaneous challenge of engaging in life while facing the imminence of death [5]. Sustaining such "double awareness" [6] is challenging and may lead to states of depression, demoralization [7], and a "psychological death" well before the literal end of life [2].

Empirically-tested, manualized group therapies for patients with advanced cancer have been found to improve psychosocial outcomes [8,9]. Group interventions are considered cost-effective as well as having the potential to offer such benefits as peer support [10], normalization of the experience [11], and modeling of successful coping strategies [12]. However, recruitment for group interventions for individuals with advanced cancer is challenging [11-15]. In addition, individual psychotherapy tends to be preferred and is often more feasible to deliver in those with advanced disease; individual sessions can be tailored to the needs of individual patients and scheduled flexibly, taking into account other hospital appointments and fluctuations in clinical status $[10,11,15$, 16]. Moreover, individual psychotherapy may be more appropriate for the discussion of intimate matters or private fears. Patients with advanced disease may need time to process these issues individually or with their partner before they can be shared in a group setting and before they feel able to absorb the fears and concerns of others in similar situations $[12,16]$.

While some manualized individual interventions included individuals with advanced cancer [17], to date, empirically-tested, manualized individual therapies that are specifically tailored to the broad range and unique needs of patients living with advanced cancer have not been available. Dignity Therapy is a brief individual therapy recently designed to address psychosocial and existential distress but is intended for patients near the end of life [3]. There is wide variability in the nature and extent of psychosocial care provided to patients with advanced disease and no widely accepted guidelines for minimal or optimal psychosocial care for this population. The lack of empirically supported manualized individual interventions contributes to this variability and to the difficulty in establishing practice guidelines [18]. To address this gap we have developed a brief, individual, manualized psychotherapeutic intervention designed to promote psychological growth and well-being and to alleviate distress in patients with advanced cancer [13]. This treatment, referred to as Managing Cancer and Living Meaningfully (CALM), was developed by a working group on psychotherapy for patients with 
advanced disease, taking into account the findings of a longitudinal program of research on the psychological well-being of patients with advanced cancer [20,21]. This paper reports findings from a qualitative study of patients participating in the pilot study of this intervention.

\section{CALM Goals and Process}

CALM is rooted in several broad theoretical traditions, including relational theory [22], attachment theory [23], and existential psychotherapy [24]. It also shares features with manualized supportive expressive [25], and meaning-centered [26] group psychotherapies applied to patients with advanced disease. The CALM intervention draws upon and complements these interventions, but is distinct from them in that it is designed as an individual therapy intended for patients with advanced disease to address the practical, relational, and existential domains of experience [19].

CALM is a semi-structured intervention that consists of 3 to 6 individual sessions, each lasting 45 to 60 minutes, delivered over 3 months, with the option of 2 booster sessions depending on clinical need. The intervention provides support and the opportunity to reflect and process thoughts and emotions evoked by the disease and its implications. It is designed both to relieve psychosocial distress and to promote psychological growth and development, a goal that has been regarded as achievable in this population [27]. CALM sessions cover four broad domains: i) symptom management and communication with health care providers; ii) changes in self and relations with close others; iii) spiritual well-being or sense of meaning and purpose; and iv) issues related to advance care planning and to end of life (thinking of the future, hope and mortality). All of these domains have been identified as common areas of concern and sources of distress in advanced cancer populations [28]. The domains provide a framework for the CALM therapist, who aims to explore them with the patient over the course of the timelimited therapy. However, the domains are often overlapping and the allocation of time to each domain is individualized, based on the concerns that are most salient or pressing to each patient.

Although CALM is an individual therapy, participation of the designated primary caregiver (usually the spouse or other family member) in one session is encouraged, allowing for more effective assessment and enhancement of family adjustment. While the first session is conducted face-to-face, follow-up sessions may be conducted over the phone when disability or geographic distance limits the ability to attend sessions at the hospital. CALM is designed to be delivered by trained mental health professionals with experience in psychosocial oncology. We have thus far trained social workers, psychologists, psychiatrists, psychiatric residents, and oncologists to deliver this intervention.

The purpose of the present study was to understand the subjective experience of advanced cancer patients receiving this new, brief individual psychotherapy and to explore its associated patient-identified benefits and risks or concerns. This information was collected prior to the initiation of a funded, randomized controlled trial testing the effectiveness of this intervention. 


\section{Method}

\section{Procedure and Sampling}

This pilot study of the CALM intervention was conducted at Princess Margaret Hospital (PMH), of the University Health Network, a large urban regional cancer center in Toronto, Canada. The study received approval from the University Health Network Research Ethics Board, and all participants provided informed written consent.

Training of CALM therapists prior to this pilot study and ongoing supervision by the clinician investigators who developed the intervention (GR and SH) was provided. Therapists were deemed competent to provide CALM after attending an initial 4-hour training session, and 15 hours of case supervision. Weekly group supervision sessions for case formulation and discussion, aimed at ensuring treatment adherence, competence and ongoing skill development continued through the treatment process.

Potential participants in CALM were identified through referrals to psychosocial services at PMH. Eligible patients were 18 years of age or older, fluent in English, had a diagnosis of advanced cancer with an expected survival of greater than 6 months, and were interested in individual psychotherapy to assist in coping with their cancer experience.

A member of the research team (EF) who was not involved in the development or delivery of the CALM therapy designed the qualitative study and collected the qualitative data. Consecutive sampling of patients who had completed at least 3 sessions of CALM therapy was employed. The researcher contacted eligible patients to explain the qualitative interview and arrange a meeting time with interested participants. Participants were interviewed on average within 2 to 3 months of their last CALM session (with a range of 2 weeks to 8 months). The consent form was reviewed with the researcher and completed prior to starting the interview. Recruitment ended when data saturation had been reached.

\section{Interviews}

Semi-structured interviews were carried out with CALM participants. Initially, participants were given the opportunity to provide a free narrative related to their diagnosis and the impact of cancer on their lives. Then they were asked about their experience of the CALM therapy and to what extent the CALM experience contributed to changes in their cancer experience. Lastly, participants were asked about the nature, quality, and impact of other support that they received during their cancer experience.

\section{Analysis}

Interviews were audio-recorded and professionally transcribed verbatim and managed using the qualitative software program Nvivo8. Transcripts were systematically coded into anticipated (namely, the four CALM domains) and emergent themes using thematic analysis [29]. This is an iterative process whereby an initial set of themes are coded and a tentative coding scheme is developed. Within each transcript, one segment of text may be coded into more than one category, and some of the text may not be deemed relevant to any category [30]. Then, the tentative codes are applied to new transcripts, and revised to adjust for new information, until no new codes emerge [29, 31]. Coding 
was conducted by one researcher $(\mathrm{RN})$, and then reviewed by other members of the research team, including the researcher who conducted the interviews (EF).

Results

Sample

Twenty-six patients were identified as eligible for CALM therapy between February, 2009 and August, 2010 and all consented to undergo CALM. These patients were all referred for psychosocial care by their oncologist for concerns including anxiety, difficulty coping, and depression.

Of those, 17 (65\%) patients completed at least 3 CALM sessions. Reasons for withdrawal of the 9 patients who did not complete 3 sessions included: geographic relocation $(n=2)$, loss of interest $(n=3)$, and death $(n=4)$. Of these 9 patients who did not complete at least 3 CALM sessions, 5 completed 2 sessions, and 4 completed 1 session.

Of the 17 patients who completed at least 3 sessions of CALM, $10(59 \%)$ patients participated in the qualitative interviews. Of the remaining 7 patients, 3 died before being approached by the researcher, 2 patients refused to participate in interviews due to declining health, and 2 patients were unreachable.

The 10 patients who participated in the qualitative interviews included 7 women and 3 men, who ranged in age from 49 to 70 years (Mean=59; SD=7.79). Eight participants self-identified as Caucasian, one as Asian, and one as Black. Eight were married and living with their spouse; 2 were single, with one living alone, and one living with adult children. The types of advanced cancer faced by these participants were diverse and included: lung ( $\mathrm{n}=2)$, Hodgkin's lymphoma, breast, ovarian, prostate, cervical, brain, sarcoma, and multiple myeloma.

Of the 10 interviewees, 6 received CALM therapy from a master's level oncology social worker, 3 from a psychiatrist, and 1 from an oncologist. Participants completed between 3 to 6 CALM sessions at the point of the interview; 4 patients were interviewed after completing 3 sessions, 3 were interviewed after 4 sessions, 1 was interviewed after 5 sessions, and 2 were interviewed after 6 sessions.

Interviews with participants ranged in duration from 45 to 90 minutes. In 2 cases, at the request of the patient, spouses were present during the interview and actively participated. Spouses who were present during the interview were asked to sign a companion consent form allowing the interview to be audio-recorded and transcribed. Interviews were conducted at a place of convenience to the patient; 6 of the interviews were conducted at the hospital, 3 were conducted over the phone, and 1 interview was conducted in the participant's home due to compromised mobility.

\section{Findings}

All participants valued the CALM intervention and indicated that it made a positive and unique contribution to their cancer experience. No participants reported any risks or concerns associated with CALM. Five interrelated themes were identified based 
on participant accounts of their CALM experience, which were: 1) a safe place to process the experience of advanced cancer; 2) the permission to talk about death and dying; 3) assistance in managing the illness and navigating the healthcare system; 4) the resolution of relational strain; and 5) the opportunity to "be seen as a whole person" within the healthcare system. These themes are discussed in detail below. Together, they capture the overall contribution of CALM to the participants' cancer experience. This contribution was unique in that it provided an experience which was not encountered anywhere else along the participants' cancer journey.

A safe place to process the experience of advanced cancer. Participants spoke of the diagnosis of advanced cancer as bringing a heightened and urgent need for reflection and re-evaluation of plans and priorities. They spoke of the need to "define what living life to the fullest" means, to "reevaluate my life and what I place emphasis on", to find meaning in life when "everything that gave you self-worth and identity is just slowly being taken away from you", and to attend to new fears and concerns regarding pain and suffering at the end of life. Participants found in CALM a "safe place" in which these issues could be "verbalized" and processed through a dialogue with a trained health professional. Participants said that without such an opportunity, it might have been too difficult and overwhelming to reflect on the experience of advanced cancer. As one participant said: "it's almost like I let myself think about it [my depressed feelings], because I knew I had a safe place to talk about it."

Participants commonly stressed that the safe, non-judgmental atmosphere of the CALM therapy was unique; all spoke of the difficulty discussing the experience of advanced cancer with family members or close friends. Participants described the reluctance to confide in others as emerging out of their wish to "protect" and to avoid "burdening" their loved ones with this new reality, and from their sense that their illness made others "uncomfortable", "afraid", or want to "pull away." By contrast, they found that CALM therapy was an "arena for venting", in which they could "dump" and "unload" their thoughts and feelings freely, and let "the floodgates open up." As one participant described:

"I guess the thing that I liked most about it [CALM] was that I could go and sort of whine if I wanted to, to somebody who was not a friend and not a family member... a person that I could go talk to and say whatever I wanted and not feel that I was going to put a burden on a friend or a family member. That was the most important thing to me. I'd say whatever I wanted, whatever I was concerned about, that was okay" (Participant \#7)

The sense of a safe space and time for reflection and communication was supported by the perception that the therapist is "a professional that's seen this before." The ability to speak with someone who is "knowledgeable about cancer" and who can "empathize in a very grounded, rooted way" added to the feeling of being understood and helped "normalize" emotions, physical symptoms, and concerns. Moreover, the opportunity to express emotions and concerns within a safe, non-judgmental environment allowed participants to gain new understanding, and to "work through" difficult emotions. In relation to this aspect of therapy, participants highlighted the importance of the therapist "steering me to go deeper" and asking "concrete and relevant questions" 
while still being "gentle", "not intrusive", and "[not] trying to take me down any paths that I don't want to go."

The ability to validate, normalize, and work through emotions in therapy was considered by participants to bring them a greater sense of peace and ability to cope. As one participant said:

"It [CALM] is certainly making me more able to deal with the cancer. I think it's helping me deal more effectively in all areas of my life...My life is better knowing I have a safe place for these things, which I didn't necessarily have before" (Participant \#6)

Processing emotions was also associated with a sense of "growth as a person" and a greater capacity to face impending death. As one participant said:

"I have to prepare myself for death, and she [the therapist] is managing to [help me] because feeling that I'm able to grow as a person, makes me feel like I will be able to handle death in a peaceful way" (Participant \#5)

The permission to talk about death and dying. Participants felt that there was an unspoken taboo about raising the topic of death and dying with friends and family, although this issue was what often concerned them the most. CALM was the only place in which a discussion of death and dying was encouraged and experienced as "part of the agenda." The opportunity for this subject to emerge openly was experienced by them as a "huge relief." Moreover, the chance to talk about death and dying in therapy helped patients to overcome their fears of raising this issue with loved ones and to initiate muchdesired open discussions with them:

"When I spoke about death, my mom would get very upset...she didn't want to hear it and I needed to talk about it... [after talking to my therapist] I told my mom that I'm not afraid of dying. I said, 'you know, Mom, with my sickness, anything is possible so we have to talk about it whether you want to hear it or not.' So now she's open whenever I'm talking about things like that, she will sit down and listen.... and I'm not afraid to talk anymore" (Participant \#2)

Assistance in managing the illness and navigating the healthcare system. The cancer-related expertise of the therapists allowed them to assist patients in managing the illness, its symptoms and side-effects, and in navigating the healthcare system. Participants reported many specific examples of such assistance, including receiving guidance on how to resolve financial issues affecting treatment and caregiving, and to arrange wills and power of attorney. In the excerpt below, one participant recalled the help the therapy provided her in assisting her young child adapt to her illness:

"[CALM] gives you the opportunity to have somebody there that's also a resource...for instance, at the beginning I was concerned about my son. How I would be able to tell him I had cancer? So in that respect the therapist was very good because she provided me with literature and she gave me ideas on how to deal with it so that was a big help, actually that was a huge help" (Participant \#1) 
This help was not only of practical benefit but its provision was emotionally meaningful within the therapeutic relationship, serving to enhance the bond with the therapist. Also, this assistance often had an impact on more than one life domain. For example, as captured by the excerpt below, the therapist helped one patient and his spouse to understand that his aggression and irritability may have been a side-effect of steroid medication. This information helped them to cope together with the "steroid storm" that had been so disturbing to both of them, and restored their relationship:

"One of the things that he [therapist] really has helped both of us with is the side effects of steroids. He really helped us so much because the steroids really have a negative effect on [my husband] as far as his personality. We shared that with him and he helped us to understand that that's a perfectly normal thing to happen, and helped us to deal with it. We've always had a wonderful, really close relationship, but it was not good for the last year and a half because I was never used to this side of [my husband] that was coming out because of the side effects, so he [therapist] walked us through what he called the 'steroid storm"' (Spouse of Participant \#9)

The resolution of relational strain. A major struggle for participants brought on by the diagnosis of advanced cancer was the management of relationships with family members and their wider social circle. Participants felt that although those around them wanted to support them, it was often difficult for their loved ones to support them in ways that they experienced as helpful. For example, one participant reported that her husband "is so worried what the chemo's going to do to me, that instead of communicating and giving me that support, he doesn't communicate at all." In this regard, participants valued the opportunity to bring family members to CALM sessions and to utilize these sessions to communicate with them in new and effective ways. For example, one participant said that a joint CALM session allowed both her and her husband to "be distraught together" without increasing each other's distress for the first time, because of the presence of the therapist who "was a grounding, safe person that we knew was there."

In addition to the impact on their relationships with spouses, participants described CALM as helping them better understand and navigate the reactions of others to their diagnosis:

"[Cancer] is recalibrating my relationship with friends, or renegotiating...there are people who desperately want to be part of this because they want to help. And there are people who are very uncomfortable with this and don't even want to talk about it and then there are people that may want to help but I actually don't want them to help. So there's the working your way through that...the therapy helped me let other people be who they are. I can choose not to be around someone whose reaction to this makes me uncomfortable...they're not a bad person because it makes me uncomfortable, there's no reason that they should know any better, it's just what it is" (Participant \#6)

The opportunity to "be seen as a whole person" within the healthcare system. Participants described forming a strong bond with their therapists. They experienced the therapists as providing "understanding, warmth, kindness", as individuals they could trust 
and who "stood by me", and who relate to them first and foremost as "a whole person." In the context of a fragmented healthcare system in which they needed to be seen by different specialists, participants also appreciated the opportunity to have several sessions with the CALM therapist, and that they were able to maintain contact with their therapists after the termination of the intervention:

"I found it helpful when we parted that she [therapist] said the door is open, if you run into an issue that, you know, I can't handle I could come back. So the door is not slammed in my face... So I felt that, you know, if the need arises I can come back...it's like a relief valve" (Participant \#10)

The sense of "being heard" and "being seen" within the medical system, as a result of CALM, was profound. Participants regarded it as an experience which was not encountered anywhere else in their cancer care, and felt that it uniquely added to their experience of their care:

"I think it's really important to provide funding for this kind of care and I think I'm probably more aware of it just because my husband has had cancer, heart surgery, and another kind of cancer. He has so many doctors and nobody puts it together. Everybody kind of looks at him as: "there's a lung problem and there's a bone problem, and there's a skin problem", so everybody is solving these little problems and nobody is looking at him as a person, and this therapy has been the only opportunity for us to be looked at as people by the medical system. And I think that that is really important because you are more than the sum of your parts. ... certainly you need to fund drugs and everything else but somewhere along the line there also has to be the type of funding that looks at who people are in their entirety and that's what a program like this does for people like my husband and myself"' (Spouse of Participant \#4)

\section{Discussion}

The subjective experience of CALM, a brief psychotherapy for patients with advanced cancer, was explored in a qualitative study of 10 patients who underwent at least 3 sessions of CALM during the pilot study of this intervention. The findings from this study demonstrate that CALM therapy was associated with profound and unique patient-identified benefits and no patient-identified risks or concerns. The positive effect of CALM was attributed by participants to the opportunity it gave them for communication and reflection, to assistance with practical problem-solving, and for the support it provided them in addressing the complex and distressing issues that the cancer had evoked within themselves and their family members. CALM was a unique experience in that the participants did not receive this kind of profound and practical experience anywhere else along their cancer journey.

Participants in the CALM therapy valued having a time and place to discuss disturbing and potentially frightening matters related to their disease, their treatment and their prognosis. The importance of this safe place for them was underscored by the 
urgency they felt to address life priorities and existential issues brought on by the diagnosis of advanced cancer. This sense of urgency was also identified in previous studies on the experience of individuals with advanced cancer [32, 33]. Also, despite feeling that their family cared about them, participants often felt isolated by their desire to protect their loved ones from the emotionally distressing implications of their condition [32-35]. Participants reported that the opportunity to talk freely in the CALM sessions provided emotional relief, greater understanding of their thoughts and feelings, and a sense of peace and personal growth. The latter is a goal that has been considered achievable in this population [27].

Our findings highlight important nonspecific therapeutic attributes of the CALM therapist. These include the nonjudgmental and empathic posture of the therapist and support for the reflective process. With regard to the latter, patients reported valuing the therapist's "insider's" perspective, which facilitated problem-solving and helped patients to feel that their experience could be more readily understood. This served to substantiate that CALM can be taught to and provided by a variety of oncology professionals as long as they have some previous training in psychotherapeutic principles, and can take an empathic and sensitive stance with their patients. We believe that a strength of the CALM intervention is that therapists from multiple disciplines can be trained to deliver CALM therapy as long as the integrity of the therapy is continuously monitored. While there may be discipline-specific differences amongst therapists in cancer-related knowledge, there is also much commonality amongst therapist in this respect, and the CALM intervention does not fundamentally vary based on the provider's expertise.

It is possible that our free narrative interview strategy resulted in findings that highlight more nonspecific therapeutic attributes than specific ones and that explicit interview questions about the domains that the CALM intervention aims to target could have revealed more CALM-specific benefits. Having said that, the general therapeutic attributes that we identified have also been reported by others in regard to psychotherapeutic interventions with cancer patients [36, 37]. For example, in a qualitative study that compared between the experiences of cognitive-behavioural therapy versus relaxation therapy for cancer patients, it was reported that the element that patients found most helpful was identical across the two approaches, namely, the therapist's ability to respond in a caring and empathic manner to their concerns [35].

Patients in CALM therapy reported appreciating the flexible structure of the therapy, which respected their individual needs regarding the pacing and content of the sessions, and the opportunity in CALM to address both practical and existential issues. That combination of the practical and the profound is central to CALM, and has been recognized by others as an important attribute of psychotherapy in the context of cancer. For example, Boulton et al. [38] reported that although patients served by psychooncology services appreciated the opportunity to express feelings and to reflect, their most common source of dissatisfaction with counseling in this context was that it was too abstract and not practical enough. Notably, our analysis suggests that the provision of practical support in sessions was not only perceived as valuable in its own right but that it also enhanced the depth of the therapeutic relationship. The nature of this practical support may vary depending on the needs of the patients and the discipline-specific knowledge of the therapist. 
There was a reverberating benefit of CALM in that it empowered participants to discuss issues with their families or close friends that they had previously found difficult to address. In particular, participants found the opportunity to discuss mortality-related concerns in the CALM sessions to be uniquely valuable and relieving, and that it facilitated subsequent conversations with family members. Indeed, we [21] and others [38] have observed that silence in families about end-of-life issues is common in the context of advanced cancer and problematic for patients in this circumstance. As has been noted in group interventions, the opportunity to discuss death and dying in the safety of therapy helps to reduce the associated anxiety and sense of isolation [39]. Furthermore, our findings suggest that CALM has the potential to facilitate relational growth and improve the ability of patients and their family members to support each other. This is noteworthy given the negative impact of advanced cancer on family caregivers' psychological well-being [40, 41] and marital functioning [41]. All participants in our study utilized the option to invite a spouse or family member to participate in CALM sessions, and perceived the joint sessions as uniquely beneficial. This finding demonstrates that optimal psychosocial care for individuals with advanced cancer must take into account the patient-caregiver unit $[42,43]$.

Lastly, the private nature of the conversation and the individual relationship with the therapist was emphasized by all participants as being important in the perceived benefit of CALM. This is consistent with the findings that a positive therapeutic relationship is associated with beneficial psychotherapy outcomes in both non-medical populations [44], and in cancer patients [9, 35, 37, 45]. In the context of advanced cancer and a busy medical setting, a meaningful relationship was recognized by participants as rare and unique. In contrast to many other interactions with health care providers in the cancer setting, the CALM intervention allowed participants to feel seen as whole persons within the healthcare system. In this way, CALM appeared to act as an antidote to the sense of dehumanization and compartmentalization that can affect both patients and health care providers in high volume medical settings that treat seriously ill patients [46].

While we believe that all advanced cancer patients who are interested in psychotherapeutic support may benefit from CALM, resource constraints may enable only those who are distressed to be offered this intervention. Consequently, our current randomized controlled trial testing the effectiveness of this intervention aims to demonstrate a reduction in depressive symptoms and other distress markers in the intervention group compared to usual care. Toward this end, recruitment strategies in the randomized controlled trial involve selectively recruiting participants based on their depression scores on routine distress screening conducted in oncology outpatient clinics, rather than based on referrals to psychosocial care, as in the present pilot study. The present qualitative study was an important first step prior to the implementation of the randomized controlled trial [47]. By highlighting patient-identified benefits of CALM, this study informed the therapeutic intervention by emphasizing the importance of elements such as empathy, pacing, the emotional meaning of practical support, and inquiry into dying and death-related concerns. Further, it served to confirm the relevance of secondary outcome measures chosen for the randomized controlled trial, including measures of post-traumatic growth, spiritual well-being, relationship quality, and distress related to dying and death. 
There are a number of limitations to the present study. The participants were all English-speaking patients receiving care in a large urban comprehensive cancer center and so may not be representative of the general population of cancer patients. Further, the participants were selected cases identified for referral to a psychosocial oncology clinical service and therefore may not be representative of a broader cross-section of patients with advanced disease. While this limitation may have biased our findings towards a positive evaluation of CALM, the data helped us identify the elements of CALM that patients find to be most helpful. Some of the limitations of the present study will be overcome in the large randomized controlled mixed-method trial which is now underway.

\section{Conclusion}

Although psychosocial care has been regarded as central to palliative and supportive care, there have been few empirically-tested approaches to individual intervention. CALM is unique in that it is a brief, semi-structured, manualized individual intervention tailored to the needs of patients with advanced disease, addressing the multiple practical, relational and existential challenges that both they and their families face. The qualitative findings reported here regarding this novel psychotherapeutic intervention are most encouraging, suggesting that it provides benefit for patients at a uniquely vulnerable time in their lives.

An intervention such as CALM has the potential to preserve quality of life and psychological well-being at a critical time before disease progression limits mobility and social engagement. The psychological state of flux produced by the diagnosis or progression of disease and by the threat of mortality may trigger not only psychological distress but also a greater openness to psychological help. An intervention such as CALM may give voice to what might otherwise be unspeakable and may diminish the sense of isolation that may affect those facing progressive disability and decline. Such approaches which are manualized and clinically relevant, can be taught, disseminated, and can contribute to the "search for meaning, well-being and self-care" [46] that may be important for patients who face the suffering and threat of mortality associated with advanced disease.

\section{Acknowledgements}

We thank our study participants and our research staff and colleagues who contributed to this project, especially Leanne Ferreira and Judy Jung for their assistance with participant recruitment and study management for the CALM pilot study. This research was supported in part by a University Health Network Department of Psychiatry Research Grant for the CALM pilot study (SH); by the Edith Kirchmann Fellowship at Princess Margaret Hospital, Toronto, Canada (EF); and by the Canadian Institutes of Health Research (CIHR) Grant \#MOP106473, October 2010-September 2015 (GR and $\mathrm{SH})$.

Role of the funding source 
The sponsors of this study had no role in study design, data collection, data analysis, interpretation of findings, or writing of this report.

\section{Conflict of interest statement}

None declared. 


\section{References}

[1] Rodin G. Treatment of depression in patients with cancer, Lancet 2008; 372: 8-10.

[2] Rodin G. Individual psychotherapy for the patient with advanced disease. In Handbook of Psychiatry in Palliative Medicine, Second Edition. Chochinov HM \& Breitbart W (Eds). London: Oxford University Press, 2009; 443-453.

[3] Chochinov HM, Hack T, Hassard T, Kristjanson LJ, McClement S, \& Harlos M. Dignity therapy: A novel psychotherapeutic intervention for patients near the end of life. Journal of Clinical Oncology 2005; 23: 5520-5525.

[4] Hales S, Zimmermann C, \& Rodin G. The quality of dying and death. Archives of Internal Medicine 2008; 168: 912-918.

[5] Lynn J. Living long in fragile health: The new demographics shape end of life care. Improving End of Life Care: Why Has It Been So Difficult? Hastings Center Report 2005; 35: S14-S18.

[6] Rodin G, \& Zimmermann C. Psychoanalytic reflections on mortality: A reconsideration. Journal of the American Academy of Psychoanalysis \& Dynamic Psychiatry 2008; 36: 181-196.

[7] Clarke DM, Kissane DW, Trauer T, \& Smith GC. Demoralization, anhedonia and grief in patients with severe physical illness. World Psychiatry 2005; 4: 96-105.

[8] Goodwin PJ, Leszcz M, Ennis M, Koopmans J, Vincent L, Guther H, Drysdale E, Hundleby M, Chochinov HM, Navarro M, Speca M, \& Hunter J. The effect of group psychosocial support on survival in metastatic breast cancer. New England Journal of Medicine 2001; 345: 1719-1726.

[9] LeMay K, \& Wilson KG. Treatment of existential distress in life-threatening illness: A review of manualized interventions. Clinical Psychology Review 2008; 28: 472493.

[10] Clark MM, Bostwick JM, \& Rummans TA. Group and individual treatment strategies for distress in cancer patients. Mayo Clinic Proceedings 2003; 78: 15381543.

[11] Moyer A, Sohl SJ, Knapp-Oliver, SK, Schneider S, \& Floyd AHL. Lessons to be learned from 25 years of research investigating psychosocial interventions for cancer patients. The Cancer Journal 2009; 15: 345-351.

[12] Edelman S, Bell DR, \& Kidman AD. A group cognitive behaviour therapy programme with metastatic breast cancer patients. Psychooncology 1999; 8: 295305.

[13] Boonzaier A, Pollard A, Ftanou M, et al. The practical challenges of recruitment and retention when providing psychotherapy to advanced breast cancer patients. Supportive Care in Cancer 2010; 18: 1605-1613. 
[14] Ford MF, Jones M, Scannell T, et al. Is group psychotherapy feasible for oncology outpatient attenders selected on the basis of psychological morbidity? British Journal of Cancer 1990; 62: 624-626.

[15] Sherman AC, Pennington J, Latif U, Farley H, Arent L, \& Simonton. Patient preferences regarding cancer group psychotherapy interventions: A view from the inside. Psychosomatics 2007; 48: 426-432.

[16] Lee V, Cohen SR, Edgar L, Laizner AM, \& Gagnon AJ. Meaning-making and psychological adjustment to cancer: Development of an intervention and pilot results. Oncology Nursing Forum 2006; 33: 291-302.

[17] Strong V, Waters R, Hibberd C, et al. Management of depression for people with cancer (SMaRT oncology 1): A randomised trial. Lancet 2008; 372: 40-48.

[18] Ellwood AL, Carlson LE, \& Bultz BD. Empirically supported treatments: will this movement in the field of psychology impact the practice of psychosocial oncology? Psychooncology 2001; 10: 199-205.

[19] Hales S, Lo C, \& Rodin G. Managing Cancer and Living Meaningfully (CALM) Treatment Manual. An Indivdual Psychotherapy for Patients with Advanced Cancer. Toronto, Canada (C February 2010).

[20] Lo C, Zimmermann C, Rydall A, Walsh A, Jones JM, Moore MJ, Shepherd FA, Gagliese L, \& Rodin G. Longitudinal study of depressive symptoms in patients with metastatic gastrointestinal and lung cancer. Journal of Clinical Oncology 2010; 28: 3084-3089.

[21] Nissim R, Gagliese L, \& Rodin, G. The desire for hastened death in individuals with advanced cancer: A longitudinal qualitative study. Social Science \& Medicine 2009; 69: 165-171.

[22] Mitchell SA. Relational Concepts in Psychoanalysis: An Integration. Cambridge, MA: Harvard University Press 1988.

[23] Bowlby J. Attachment. New York: Basic Books 1982.

[24] Yalom ID. Existential Psychotherapy. New York, NY: Basic Books 1980.

[25] Spiegel D, Morrow GR, Classen C, Raubertas R, Stott PB, Mudaliar N, Pierce HI, Flynn PJ, Heard L, \& Riggs G. Group psychotherapy for recently diagnosed breast cancer patients: A multicenter feasibility study. Psycho-Oncology 1999; 8: 482-493.

[26] Breitbart W, Gibson C, Poppito SR, \& Berg A. Psychotherapeutic interventions at the end of life: A focus on meaning and spirituality. Canadian Journal of Psychiatry 2004; 49: 366-372.

[27] Block SD. Perspectives on care at the close of life. Psychological considerations, growth, and transcendence at the end of life: The art of the possible. JAMA 2001; 285: 2898-2905.

[28] Kissane DW, Levin T, Hales S, Lo C, Rodin G. Psychotherapy for depression in cancer and palliative care. In Depression and Cancer, Kissane D, Maj M, \& Sartorius N (Eds). Chichester, UK: Wiley 2010; 177-206. 
[29] Luborsky M. The identification and analysis of themes and patterns. In Qualitative Methods in Aging Research, Gubrium JF, \& Sankar A (Eds.). Thousand Oaks, CA: Sage Publications 1994; 189-210.

[30] Thomas D. A general inductive approach for analyzing qualitative evaluation data. American Journal of Evaluation 2006; 27: 1-10.

[31] Huberman AM, \& Miles MB. Data management and analysis methods. In Handbook of Qualitative Research, Denzin NK, \& Lincoln YS (Eds.). Thousand Oaks, CA: Sage Publications 2004; 413-427.

[32] Berterö C, Vanhanen M, \& Appelin G. Receiving a diagnosis of inoperable lung cancer: Patients' perspectives of how it affects their life situation and quality of life. Acta Oncologica 2008; 47: 862-869.

[33] Nissim R, Rennie D, Fleming S, Hales S, Gagliese L, \& Rodin G. Goals at the end of life: Findings from a qualitative longitudinal study with individuals with fatal cancer. Death Studies (In press).

[34] Hjorleifsdottir E, Hallerg IR, Gunnarsdottir ED, \& Bolmsjo IA. Living with cancer and perception of care: Icelandic oncology outpatients, a qualitative study. Supportive Care in Cancer 2008; 16: 515-524.

[35] MacCormack T, Simonian J, Lim J, Remond L, Roets D, Dunn S, \& Butow P. 'Someone who cares:' A qualitative investigation of cancer patients' experiences of psychotherapy. Psycho-Oncology 2001; 10: 52-65.

[36] Donnelly JM, Kornblith AB, Fleishman S, Zuckerman E, Raptis G, Hudis CA, Hamilton N, Payne D, Massie MJ, Norton L, \& Holland JC. A pilot study of interpersonal psychotherapy by telephone with cancer patients and their partners. Psycho-Oncology 2000; 9: 44-56.

[37] Manne S, Winkel G, Zaider T, Rubin S, Hernandez E, \& Bergman C. Therapy processes and outcomes of psychological interventions for women diagnosed with gynecological cancers: A test of the generic process model of psychotherapy. Journal of Consulting and Clinical Psychology 2010; 78: 236-248.

[38] Boulton M, Boudioni M, Mossman J, Moynihan C, Leydon G, \& Ramirez A. "Dividing the desolation": Clients' views on the benefits of a cancer counselling service. Psycho-Oncology 2001; 10: 124-136.

[39] Spiegel D. Essentials of psychotherapeutic intervention for cancer patients. Supportive Care in Cancer 1995; 3: 252-256.

[40] Braun, M., Mikulincer, M., Rydall, A., Walsh, A., \& Rodin, G. The hidden morbidity in cancer: Spouse caregivers. Journal of Clinical Oncology 2007; 25: 4829-4834.

[41] Northouse, L.L.,Templin,T.,Mood,D., et al. Couples' adjustment to breast cancer and benign breast disease: A longitudinal analysis. Psycho-Oncology 1998; 7: 3748. 
[42] Northouse, L. L., Katapodi, M., Song, L., Zhang, L., Mood, D.W. Interventions with family caregivers of cancer patients: Meta-analysis of randomized clinical trials. Ca: A Cancer Journal for Clinicians 2010; 60: 317-339.

[43] Mclean, L. M., \& Nissim, R. Marital therapy for couples facing advanced cancer: Case review. Palliative and Supportive Care 2007; 5: 303-313.

[44] Norcross JC. Psychotherapy Relationships that Work: Therapist Contributions and Responsiveness to Patients. New York, NY: Oxford University Press 2002.

[45] Schnur JBG, \& Montgomery GH. A systematic review of therapeutic alliance, group cohesion, empathy, and goal consensus/collaboration in psychotherapeutic interventions in cancer: Uncommon factors? Clinical Psychology Review 2010; 30: 238-247.

[46] Cole TR, \& Carlin N. The suffering of physicians. The Lancet 2009; 374: 14141415.

[47] Lancaster GA, Dodd S, \& Williamson PR. Design and analysis of pilot studies: Recommendations for good practice. Journal of Evaluation in Clinical Practice 2004; 10: 307-312. 